\title{
Anger, Quality of Life and Mood in Multiple Sclerosis
}

\author{
Christianne M. Laing ${ }^{1 *}$, Louise H. Phillips ${ }^{1}$, Clare. L. Cooper ${ }^{1}$, Judith A. Hosie ${ }^{1}$ and Fiona Summers ${ }^{2}$ \\ ${ }^{1}$ University of Aberdeen, Aberdeen, Scotland, UK \\ ${ }^{2}$ Aberdeen Royal Infirmary, Foresterhill, Aberdeen, UK \\ *Corresponding author: Christianne M. Laing, University of Aberdeen, Aberdeen, Scotland, UK, Tel: 441224 273522; E-mail: r02cml12@abdn.ac.uk
}

Received date: Sep 02, 2014, Accepted date: Oct 28, 2014, Publication date: Nov 05, 2014

Copyright: ( 2014 M. Laing C, et al. This is an open-access article distributed under the terms of the Creative Commons Attribution License, which permits unrestricted use, distribution, and reproduction in any medium, provided the original author and source are credited.

\begin{abstract}
Objective: To investigate the role of different anger variables in predicting quality of life and mood in multiple sclerosis (MS), while controlling for disease severity.

Methods: 78 individuals with MS completed questionnaire measures of anger (Anger Rumination Scale and State/Trait Anger Expression Inventory), mood (Hospital Anxiety and Depression Scale); disease severity (Patient Determined Disease Steps) and quality of life (WHOQoL-BREF).
\end{abstract}

Results: Anger was significantly associated with quality of life (QoL) in MS. Higher levels of experienced anger and lack of control over the experience of anger predicted lower psychological and environmental QoL. Depression levels were also predicted by high levels of experienced anger and lack of control over anger. In contrast, ruminating about anger was the strongest predictor of anxiety levels.

Conclusions: The results demonstrate that feelings of anger are strongly associated with QoL in MS, even when disease severity is taken into account. Problems in controlling and ruminating about anger may also contribute to mood disorder in MS.

Keywords: Anger; Rumination; Depression; Anxiety; Quality of Life; Multiple Sclerosis

\section{Introduction}

Multiple Sclerosis (MS) is a chronic demyelinating disease of the central nervous system that causes various neurological impairments. As well as the health challenges faced by those with MS, the disease is also associated with emotional disturbances. Depression, anxiety, anger and irritability are frequently reported in MS patients [1-4]. People with MS often report feelings of anger and irritation, and anecdotally, this appears to negatively impact their overall quality of life (QoL) and increases feelings of depression and anxiety. MS patients often report reduced QoL compared, not only to the general population, but also patients with other chronic diseases [5]. Yet few studies have examined the role of anger in QoL and mood disorder in individuals with MS, therefore that is the focus of the current research.

Anger is one of the most frequently experienced negative emotions [6] and can have serious negative effects on everyday life [7]. Expressing anger is specifically associated with interpersonal difficulties, such as social maladjustment, while expressing other negative emotions such as fear and sadness are not $[8,9]$. However, evidence suggests uninhibited expressions of anger, or chronic suppression of anger, may also be detrimental to an individual's physical health [10-12], social involvement $[13,14]$ and close personal relationships [15-17]. Moreover, anger inducing situations often lead to rumination, i.e. recurrent thoughts about the causes and consequences of the event, which have also been shown to have maladaptive consequences, such as elevated blood pressure [18] and an increase in depressed mood [19]. Therefore it is apparent that anger has negative consequences both physiologically and psychologically for individuals, yet this could be a greater risk in clinical populations where feelings of anger may be more heightened.

Heightened anger has been observed amongst clinical populations with similar disease progression to MS, including arthritis [20,21], fibromyalgia [22] and chronic pain [23,24]. The frustrations of people suffering from such illnesses, such as persistent bodily complaints, and limited feedback on the aetiology and progression of their illness [23] are likely to be similar for individuals with MS. In addition to the heightened anger associated with the reactive response to the illness, brain specific lesions in MS might also influence the experience and control of emotions such as anger [25]. As anger has been associated with depressed mood [19] and there is a lifetime prevalence of depression in MS as high as 50\% [26-28], understanding links between anger and depression in MS is important.

Currently there is a paucity of research investigating anger and MS in any depth. Nocentini and colleagues [1] assessed anger in 195 participants with MS, in relation to demographic, clinical and mood characteristics. Anger was assessed using the State Trait Anger Expression Inventory (STAXI). Their results suggested higher levels of withheld anger and lower levels of controlled anger than might be expected in the general population, independent of disease severity. Significant associations between anger, anxiety and depression were reported. Consequently, there appears to be some evidence that, not only is anger experienced more in MS than in the general population, but anger is significantly associated with negative affect. However this study did not explore the issue of anger rumination, which is likely to be particularly important in the development of anxiety and depression. Also to date, there appear to be no studies examining how 
anger experience and control influences QoL in people with MS Therefore, this study will examine the link between anger experience/ control and QoL in people with MS. Given the very individual nature of MS, it is likely that some people with the condition will not experience unusual levels of anger, whilst others experience serious problems with this emotion, and the current study aims to explore this. By using multidimensional assessments of anger we will explore which aspects of anger are most important in predicting QoL. The STAXI-2 [29] separates out the different processes which might be involved in the internal experience of anger (AX-I) from the outward expression of anger through aggression (AX-O). It also assesses how people control their anger: whether through internal control strategies such as counting to ten (AC-I), or through the active suppression of outward expression (AC-O). Our other assessment of anger is the Anger Rumination Scale [30] which measures the extent to which people ruminate about feelings of anger or frustration.

The possible links between these different aspects of anger and QoL will be explored. In the current study we used the WHOQoL-BREF [31] which assesses four sub-domains of self-reported QoL: physical (e.g. energy levels), psychological (e.g. self-esteem), social (e.g. personal relationships) and environmental (e.g. finances). The WHOQoL-BREF identifies psychosocial effects of MS which might otherwise go unnoticed, and there is evidence of good validity of this measure in MS populations (in terms of substantial correlations with depression and disability, as well as caregiver assessments of QoL) [32]. It was hypothesised that people with MS who reported experiencing feelings of anger or lack of control over their anger would also report lower QoL. We used regression analyses to explore which aspects of anger were the strongest predictors of QoL, taking into account individual differences in disease severity. We also explored the links between anger experience and control and mood in MS by assessing anxiety and depression levels.

\section{Method}

\section{Participants}

300 participants from the NHS Grampian MS database were contacted, 85 of whom gave their consent ( $28 \%$ response rate). Seven were excluded: 5 due to incomplete data and 2 due to substance abuse, yielding a sample of 78 participants (64 female, mean age $=46.0$; $\mathrm{SD}=10.06$; range 24-68 years). Mean years education was 14.77 years $(\mathrm{SD}=3.33)$. Mean disease duration was 11.18 years $(\mathrm{SD}=7.09)$. Fifty participants had the relapsing-remitting form of the disease, 21 participants had the progressive type, whilst for 7 participants disease type was not recorded.

\section{Materials and Procedure}

Participants were sent questionnaire packs containing the following measures: World Health Organisation Quality of Life Questionnaire (WHOQoL-BREF) [31]. This required participants to rate aspects of their QoL over the previous two weeks. Four distinct domains of QoL (physical, psychological, social, environmental) were assessed using 24 questions. Higher scores represent higher QoL, with scores calculated from 0-100.

Anger Rumination Scale (ARS): This 19-item scale measures the tendency to focus on angry episodes, recall past anger experiences and think about causes and consequences of anger. It was developed to assess the cognitive processes that unfold after the emotion of anger has been triggered. Here, we combined the four domains of the ARS (Angry Afterthoughts, Thoughts of Revenge, Angry Memories and Understanding of Causes) to form one total score. Higher scores represent a greater overall tendency to focus on angry moods, recall past anger episodes, and think over the causes and consequences of anger episodes [30].

State/Trait Anger Expression Inventory-2 (STAXI-2): This questionnaire comprises 57 items measuring anger experience and control on six subscales: State Anger: the intensity of feeling; Trait Anger: anger as a personality trait; Anger Expression-Out: the tendency to express the anger externally; Anger Expression-In: the tendency to experience anger internally but not show it externally; Anger Control-Out: the control of external anger expression and Anger Control-In: the control of internalised anger. High scores on the first four sub-scales demonstrate a greater tendency to experience or express anger, whereas high scores on the latter two sub-scales (Anger Control In and Out) demonstrate an increased tendency towards controlling anger. In the present study all scales were administered (totalling 41 items) with the exception of the State Anger scale; as pilot work in the department has indicated that it revealed floor effects [33]. The STAXI-2 has been found to be a valid, reliable measure of the constructs it was intended to represent [29].

Hospital Anxiety and Depression Scale (HADS): This well validated 14-item measure is used to assess current levels of anxiety and depression. Individuals are asked to indicate which response comes closest to how they have been feeling in the past week. Higher scores indicate the increased presence of anxiety and/or depression [34].

Patient Determined Disease Steps (PDDS): This measure assesses disease progression in MS on an 8 item scale from 'Normal' through to 'Confined to wheelchair'. The PDDS has been found to be userfriendly, produce evenly distributed scores and correlate highly with the clinician assessed Kurzke Expanded Disability Status Scale (EDSS) [35-38].

\section{Results}

Table 1 shows descriptive statistics and correlations between dimensions of anger (STAXI and ARS) and QoL. Higher levels of trait anger were associated with lower perceived QoL across all domains, with the exception of physical QoL. Those who expressed higher levels of anger also reported lower psychological, social and environmental QoL, regardless of whether anger was experienced inwardly (Anger Exp. In) or expressed outwardly (Anger Exp. Out). Poor physical QoL was associated with experiencing anger inwardly, but not expressing it outwardly. Conversely, high levels of control over anger correlated positively with QoL across all domains, with the exception of physical QoL. Control of the inner experience of anger (Anger Control In) and control of the outward display of anger (Anger Control Out) were associated with good psychological and environmental QoL. However, only the control of outward anger was significantly associated with social QoL. 
Citation: Laing CM, Phillips LH, Cooper CL, Hosie JA, Summers M (2015) Anger, Quality of Life and Mood in Multiple Sclerosis. J Mult Scler 2:

Page 3 of 6

\begin{tabular}{|c|c|c|c|c|c|c|}
\hline \multicolumn{7}{|l|}{ QoL Scale } \\
\hline & $\mathbf{M}$ & SD & Phys & Psych & Soc & Environ \\
\hline \multicolumn{7}{|l|}{ STAXI: } \\
\hline Trait Anger & 14.68 & 4.83 & -.165 & $-.509^{\star *}$ & $-.384^{* *}$ & $-.482^{* *}$ \\
\hline Anger Exp. Out & 13.42 & 3.54 & -.103 & $-.415^{* *}$ & $-.334^{* *}$ & $-.399^{* *}$ \\
\hline Anger Exp. In & 16.32 & 4.74 & $-.256^{*}$ & $-.560^{\star *}$ & $-.395^{* *}$ & $-.495^{* *}$ \\
\hline Anger Control Out & 24.67 & 5.28 & .146 & $.282^{*}$ & 178 & $.342^{* *}$ \\
\hline Anger Control In & 22.54 & 5.05 & .166 & $.345^{* *}$ & $.231^{*}$ & $.406^{* *}$ \\
\hline ARS Total & 31.27 & 11.86 & $-.314^{* *}$ & $-.558^{\star *}$ & $-.283^{*}$ & $-.457^{\star *}$ \\
\hline \multicolumn{2}{|c|}{ Note: ${ }^{*} p<.05,{ }^{* *} p<.01$} & M & 13.05 & 13.76 & 15.28 & 15.81 \\
\hline & & SD & 2.41 & 3.99 & 4.11 & 3.39 \\
\hline
\end{tabular}

Table 1: Descriptive statistics and Pearson correlations between anger variables (STAXI and ARS) and QoL dimensions.

With respect to the ARS, those who reported the tendency to ruminate on angry episodes also reported experiencing lower QoL, in particular psychological and environmental aspects. Table 2 shows the relationships of the anger measures with anxiety, depression and disease severity. Both anxiety and depression correlated highly with the anger measures, with higher levels of mood symptoms associated with greater trait anger, more expression of anger, and less control over anger. Ruminating on angry experiences was highly correlated with mood, particularly anxiety.

\begin{tabular}{|l|l|l|l|l|}
\hline & & Severity & HADS-Anxiety & HADS-Depression \\
\hline STAXI: & & & & \\
\hline Trait Anger & & $.408^{* *}$ & $.599^{* *}$ & $.502^{* *}$ \\
\hline Anger Exp. Out & & $.348^{* *}$ & $.525^{* *}$ & $.417^{* *}$ \\
\hline Anger Exp. In & & $.247^{* *}$ & $.446^{* *}$ & $.612^{* *}$ \\
\hline Anger Control Out & & $.439^{* *}$ & $-.360^{* *}$ & $-.353^{* *}$ \\
\hline Anger Control In & & $-.240^{*}$ & $-.382^{* *}$ \\
\hline ARS Total & & $.461^{* *}$ & $-.251^{* *}$ & $.558^{* *}$ \\
\hline Note: ${ }^{* *} \mathrm{p} .05,{ }^{* *} \mathrm{p}<01$. & $\mathrm{M}$ & 20.17 & $.699^{* *}$ & 11.97 \\
\hline & $\mathrm{SD}$ & 12.85 & 16.36 & 4.19 \\
\hline
\end{tabular}

Table 2: Descriptive statistics and Pearson correlations between dimensions of anger, PDDS severity measure and HADS measures of anxiety and depression.

In order to investigate whether the anger variables predicted QoL, taking into account disease severity, a series of hierarchical regression analyses were carried out (Table 3). Each measure of QoL (physical, psychological, social, environmental) was treated as a separate dependent variable. As a first step in each regression, ratings of disease severity were entered. Step two included anger rumination total scores (ARS), and the measures from the STAXI (trait anger, anger expression in and out, anger control in and out) as predictors. Note that the regression statistics indicated that multicollinearity was not present in these analyses. Disease severity was the only significant predictor of physical QoL, and the anger variables did not explain additional variance. Disease severity was also a predictor of both psychological QoL and environmental QoL. Both aspects of QoL showed a similar pattern: disease severity explained a significant portion of the variance in QoL, as did the anger variables. In particular, frequent internal experiences of anger were associated with lower reported levels of psychological and environmental QoL and greater internal control of anger was associated with higher reported levels of these aspects of QoL. While anger variables added significantly to the variance in social QoL explained by disease severity, none of the individual anger measures reached significance. 
Page 4 of 6

\begin{tabular}{|c|c|c|c|c|}
\hline & \multicolumn{4}{|c|}{ QoL Scale } \\
\hline & Physical & $\begin{array}{l}\text { Psychologic } \\
\text { al }\end{array}$ & Social & $\begin{array}{l}\text { Environment } \\
\text { al }\end{array}$ \\
\hline \multicolumn{5}{|l|}{ Predictors: Step 1} \\
\hline Severity $(\beta)$ & $-.697^{\star *}$ & $-.597^{\star *}$ & $-.335^{* *}$ & $-.593^{* *}$ \\
\hline$F(1,77)$ & $71.99^{* *}$ & $42.13^{* *}$ & $9.59^{* *}$ & $41.18^{* *}$ \\
\hline $\mathrm{R}^{2}$ & .49 & .36 & .11 & .35 \\
\hline \multicolumn{5}{|l|}{ Predictors: Step 2} \\
\hline Severity ( $\beta$ ) & $-.737^{* *}$ & $-.371^{* *}$ & -.191 & $-.430^{\star *}$ \\
\hline Rumination ( $\beta$ ) & -.113 & -.158 & .125 & -.005 \\
\hline Trait Anger ( $\beta$ ) & .302 & -.048 & -.204 & -.011 \\
\hline Anger Exp In ( $\beta$ ) & .004 & $-.250^{*}$ & -.223 & $-.217^{*}$ \\
\hline Anger Exp Out ( $\beta$ ) & -.014 & -.156 & -.226 & -.171 \\
\hline Anger Control In ( $\beta$ ) & 123 & $.284^{*}$ & .220 & $.331^{* *}$ \\
\hline $\begin{array}{l}\text { Anger Control Out } \\
(\beta)\end{array}$ & .038 & -.256 & -.299 & -.154 \\
\hline$F(6,70)$ & $11.10^{\star *}$ & $12.98^{\star *}$ & $3.66^{* *}$ & $10.86^{* *}$ \\
\hline$R^{2}$ change & .04 & $.21^{* *}$ & $.16^{*}$ & $.17^{* *}$ \\
\hline
\end{tabular}

\begin{tabular}{|l|l|l|}
\hline Anger Exp Out $(\beta)$ & .148 & .136 \\
\hline Anger Control In $(\beta)$ & -.002 & $-.268^{* *}$ \\
\hline Anger Control Out $(\beta)$ & .082 & .080 \\
\hline$F(6,70)$ & $11.22^{* *}$ & $16.23^{* *}$ \\
\hline$R^{2}$ change & $.30^{* *}$ & $.22^{* *}$ \\
\hline Note: ${ }^{*} p<.05,{ }^{* *} p<.01$. & \\
\hline
\end{tabular}

Table 4: Regression analyses for anxiety and depression as indexed by HADS. The contribution of anger variables in predicting the subscales of QoL is indicated by significant individual $\beta$-weightings, and the significance of $\mathrm{F}$ values at Step 2 in the analyses.

\section{Discussion}

These results add to the literature by indicating that anger is an important predictor of QoL in people with MS. Psychological and environmental QoL were strongly associated with everyday level of anger (as assessed by trait anger), high levels of experienced and expressed anger, lack of control over anger, and a tendency to ruminate on angering experiences. Poorer physical QoL was correlated with a tendency to experience anger inwardly and ruminate on anger. Social QoL was associated with all aspects of anger except the control of outward expression. All aspects of anger were significantly related to anxiety and depression ratings. This suggests that the tendency amongst people with MS to experience mood disturbances such feelings of anger and frustration reported in previous studies [1-4] is strongly associated with poorer overall well-being and key patient centred outcomes. The findings reported are consistent with empirical evidence revealing that people with MS report experiencing low QoL [5].

Regression analyses were used to help understand which aspects of anger were most important in predicting QoL and mood in MS, in addition to variance explained by disease severity. Results indicated that anger variables explained variance in most domains of QoL over and above the prediction from disease severity. This indicates that links between anger experience/control and QoL in MS are not driven solely by individuals simply experiencing greater levels of disease related symptoms. Use of the STAXI and Anger Rumination Scales allowed us to look in more detail at which aspects of anger were more important in predicting QoL. For both psychological and environmental QoL, the key aspects of anger were frequent internal experiences of anger and lack of ability to control this inner experience of anger. To be more specific, high levels of experienced anger were associated with lower levels of psychological and environmental QoL, whereas higher levels of internal anger control were associated with higher levels of QoL in these domains. It is notable that the internal experience and internal control over anger were more important than experiencing and controlling the outward display of anger. This suggests that internal experiences of anger might adversely impact on well-being in people with MS, while having a sense of control over angry feelings might improve QoL. This could be a potential area for future intervention in management of the disease. It was also interesting to note that, when disease severity was controlled for, rumination ceased to be a significant predictor of poor QoL. These results therefore suggest that the extent to which disease related symptoms are experienced has a greater influence on individuals' self- 
rated QoL than the extent to which they dwell on angry feelings or episodes.

Anger variables were associated with higher levels of anxiety and depression in the current sample. Regression analyses revealed that levels of depression were again predicted by frequent internal experience of angry emotions, and a lack of sense of internal control over anger. In contrast, anxiety levels were strongly predicted by the tendency to ruminate over angering experiences. This has potential implications for therapeutic interventions in MS for people who are experiencing distress related to their illness. Focusing on the inner control of anger and the tendency to ruminate on frustration could help to improve mood.

These findings extend previous research demonstrating the possible maladaptive consequences of both the suppression and expression of anger [10-17]. In the current study the internalised aspects of anger (inner experience, inner control, and rumination) were more important in predicting mood and well-being than more external aspects such as displaying emotion. One important caveat to the current study is the reliance on self- report questionnaires, which are subject to mood-related biases and anosognosia. While outward expression of anger might be assessed using relative reports, it is difficult to assess the inner experience of emotions or the control strategies used in any other way than relying on self-report.

These results build on other studies which suggest that emotional skills are likely to be important in influencing QoL in MS. Previous research indicates that problems with emotion regulation and emotion perception predict impaired QoL in people with MS [38-40]. The current results again indicate that problems with emotional skills in MS, such as experiencing and controlling anger, predict QoL, anxiety and depression, independently of current assessment of disease severity. Thus, while most measures of disease severity in MS focus mainly on physical symptoms of the disease, there is a clear need to understand more about emotional and social skills to address QoL in people with MS. One issue with all of these studies to date is that the cross-sectional nature of the research makes it difficult to draw conclusions about causality. Given the often fluctuating nature of MS symptoms, it will be an important task for future research to explore the temporal nature of the relationship between emotions and QoL in MS; perhaps through the use of experience sampling methods. Longitudinal methods might also help to tease apart how coping with intense emotional issues raised by living with a lifelong degenerative condition relate to the experience of anger. Given that the experience and control of anger might be influenced by changes in the neurocognitive systems involved in self-regulation in MS, it would also be useful in future studies to include assessments of cognitive measures, as well as brain scan information.

\section{Conclusions}

Most measures of disease severity in MS focus solely on physical symptoms of the condition. The current study indicates that frequent experiences of anger, along with a lack of control over anger contribute to QoL in MS. It is important, therefore, that emotional issues are more widely assessed and discussed by clinicians and those with MS, as they might influence well-being as much as the more widely recognised motor symptoms.

\section{Acknowledgements}

This research was funded by The Multiple Sclerosis Society (UK).

\section{References}

1. Nocentini U, Tedeschi G, Migliaccio R, Dinacci D, Lavorgna L, et al. (2009) An exploration of anger phenomenology in multiple sclerosis. Eur J Neurol 16: 1312-1317.

2. Ghaffar O, Feinstein A (2007) The neuropsychiatry of multiple sclerosis: a review of recent developments. Curr Opin Psychiatry 20: 278-285.

3. Minden SL (2000) Mood disorders in multiple sclerosis: diagnosis and treatment. J Neurovirol 6 Suppl 2: S160-167.

4. Feinstein A, Feinstein K (2001) Depression associated with multiple sclerosis. Looking beyond diagnosis to symptom expression. J Affect Disord 66: 193-198.

5. Nortvedt MW, Riise T (2003) The use of quality of life measures in multiple sclerosis research. Mult Scler 9: 63-72.

6. Averill JR (1983) Studies on anger and aggression. Implications for theories of emotion. Am Psychol 38: 1145-1160.

7. Lazarus RS (1966) Psychological stress and the coping process. New York, NY: McGraw-Hill.

8. Deffenbacher J L (1992) Trait anger: theory, findings and implications. In C. D. Spielberger \& J. N. Butcher, Advances in personality assessment 9: 177-201.

9. Kubany ES, Bauer GB, Muraoka MY, Richard DC, Read P (1995) The impact of labelled anger and blame in intimate relationship. Journal of Social and Clinical Psychology 14: 53-60.

10. Mittleman MA, Maclure M, Sherwood JB, Mulry RP, Tofler GH, et al. (1995) Triggering of acute myocardial infarction onset by episodes of anger. Determinants of Myocardial Infarction Onset Study Investigators. Circulation 92: 1720-1725.

11. Siegman AW, Smith T W (1994) Anger, hostility and the heart. Hillsdale, NJ: Lawrence Erlbaum Associates.

12. Tice DM, Baumeister RF (1993) Controlling anger: Self-in- duced emotion change. In D. M. Wegner \& J. W. Pennebaker (Eds.), Handbook of mental control. Englewood Cliffs, NJ: Prentice-Hall : 393-409.

13. van Middendorp H, Geenen R, Sorbi MJ, Hox JJ, Vingerhoets AJ, et al. (2005) Styles of emotion regulation and their associations with perceived health in patients with rheumatoid arthritis. Ann Behav Med 30: 44-53.

14. Lopes PN, Salovey P, Coté S, Beers M (2005) Emotion regulation abilities and the quality of social interaction. Emotion 5: 113-118.

15. Gross JJ, John OP (2003) Individual differences in two emotion regulation processes: Implications for affect, relationships and well-being. Journal of Personality and Social Psychology 85: 248-362.

16. Gross JJ (2002) Emotion regulation: affective, cognitive, and social consequences. Psychophysiology 39: 281-291.

17. Abel GM (2011) Different stage, different performance: the protective strategy of role play on emotional health in sex work. Soc Sci Med 72: 1177-1184.

18. Gerin W, Davidson KW, Christenfeld NJ, Goyal T, Schwartz JE (2006) The role of angry rumination and distraction in blood pressure recovery from emotional arousal. Psychosom Med 68: 64-72.

19. Rusting CL, Nolen-Hoeksema S (1998) Regulating responses to anger: effects of rumination and distraction on angry mood. J Pers Soc Psychol 74: 790-803.

20. Cobb S (1959) Contained hostility in rheumatoid arthritis. Arthritis \& Rheumatism 2: 419-425.

21. Moldofsky H, Chester WJ (1970) Pain and mood patterns in patients with rheumatoid arthritis. A prospective study. Psychosom Med 32: 309-318.

22. van Middendorp H, Lumley MA, Moerbeek M, Jacobs JW, Bijlsma JW, et al. (2010) Effects of anger and anger regulation styles on pain in daily life of women with fibromyalgia: a diary study. Eur J Pain 14: 176-182.

23. Fernandez E, Turk DC (1995) The scope and significance of anger in the experience of chronic pain. Pain 61: 165-175.

24. Okifuji A, Turk DC, Curran SL (1999) Anger in chronic pain: investigations of anger targets and intensity. J Psychosom Res 47: 1-12. 
Citation: Laing CM, Phillips LH, Cooper CL, Hosie JA, Summers M (2015) Anger, Quality of Life and Mood in Multiple Sclerosis. J Mult Scler 2:

25. Lima FS, Simioni S, Bruggimann L, Ruffieux C, Dudler J, et al. (2007) Perceived behavioral changes in early multiple sclerosis. Behav Neurol 18: 81-90.

26. Feinstein A (2011) Multiple sclerosis and depression. Mult Scler 17: 1276-1281.

27. Siegert RJ, Abernethy DA (2005) Depression in multiple sclerosis: a review. J Neurol Neurosurg Psychiatry 76: 469-475.

28. Minden SL, Orav J, Reich P (1987) Depression in multiple sclerosis. Gen Hosp Psychiatry 9: 426-434.

29. Deschênes SS, Dugas MJ, Fracalanza K, Koerner N (2012) The role of anger in generalized anxiety disorder. Cogn Behav Ther 41: 261-271.

30. Sukhodolsky DG, Golub A, Cromwell EN (2001) Development and validation of the anger rumination scale. Personality and Individual Differences 31: 689-700.

31. Skevington SM, Lotfy M, O'Connell KA (2004) The World Health Organisation's WHOQOLBREF quality of life assessment: Psychometric properties and results of the international field trial. A report from the WHOQOL group. Quality of Life Research 13: 299-310.

32. Alshubaili AF, Awadalla AW, Ohaeri JU, Mabrouk AA (2007) Relationship of depression, disability, and family caregiver attitudes to the quality of life of Kuwaiti persons with multiple sclerosis: a controlled study. BMC Neurol 7:31.
33. Phillips LH, Henry JD, Hosie JA, Milne AB (2006) Age, anger regulation and well-being. Aging Ment Health 10: 250-256.

34. Snaith RP, Zigmond AS (1994) HADS: Hospital Anxiety and Depression Scale. Windsor: NFER Nelson.

35. Hohol MJ, Orav EJ, Weiner HL (1995) Disease steps in multiple sclerosis: a simple approach to evaluate disease progression. Neurology 45: 251-255.

36. Kurtzke JF (1983) Rating neurologic impairment in multiple sclerosis: An expanded disability status scale (EDSS). Neurology 33: 1444-1452.

37. Learmonth YC, Motl RW, Sandroff BM, Pula JH, Cadavid D (2013) Validation of patient determined disease steps (PDDS) scale scores in persons with multiple sclerosis. BMC Neurol 13: 37.

38. Phillips LH, Henry JD, Nouzova E, Cooper C, Radlak B, et al. (2014) Difficulties with emotion regulation in multiple sclerosis: Links to executive function, mood, and quality of life. J Clin Exp Neuropsychol .

39. Phillips LH, Henry JD, Scott C, Summers F, Whyte M, et al. (2011) Specific impairments of emotion perception in multiple sclerosis. Neuropsychology 25: 131-136.

40. Phillips LH, Saldias A, McCarrey A, Henry JD, Scott C, et al. (2009) Attentional lapses, emotional regulation and quality of life in multiple sclerosis. Br J Clin Psychol 48: 101-106. 